\title{
Gold dimer in neon: an absorption and fluorescence study
}

\author{
S. Lecoultre ${ }^{\mathrm{a}}$, A. Rydlo, C. Félix ${ }^{\mathrm{b}}$, and W. Harbich \\ Institut de Physique des Nanostructures, EPFL, 1015 Lausanne, Switzerland
}

Received 19 September 2008

Published online 24 January 2009 - (c) EDP Sciences, Società Italiana di Fisica, Springer-Verlag 2009

\begin{abstract}
We report for the first time the absorption and fluorescence spectra of gold dimers in a neon matrix. The dimer absorption spectra show the $\mathrm{A} \leftarrow \mathrm{X}$ transition predicted from measurements in the gas phase and not observed so far in a matrix, as well as the so-called $\mathrm{B} \leftarrow \mathrm{X}$ and $\mathrm{C} \leftarrow \mathrm{X}$ transitions. Fluorescence measurements on the atom reveal new emission lines at 1.97, 3.59 and $4.09 \mathrm{eV}$ that can be assigned to the ${ }^{2} \mathrm{P}_{1 / 2} \rightarrow{ }^{2} \mathrm{D}_{3 / 2},{ }^{2} \mathrm{P}_{1 / 2} \rightarrow{ }^{2} \mathrm{D}_{5 / 2}$ and the ${ }^{2} \mathrm{P}_{3 / 2} \rightarrow{ }^{2} \mathrm{D}_{5 / 2}$ transitions. For the dimer, excitation of both $\mathrm{A}$ and $\mathrm{B}$ state results in distinct emission spectra with vibrational structure.
\end{abstract}

PACS. 36.40.Vz Optical properties of clusters

\section{Introduction}

One of the objectives of the field of cluster science is to understand the evolution of the properties of matter as a function of the number of atoms. In this respect the dimer has a special interest, since it is the first building block in which the nature of the bonding between atoms appears. Furthermore a detailed comparison between theory and experiment is possible due to the small number of atoms involved.

Optical spectroscopy is a powerful technique to investigate the electronic properties of small clusters. However, measurements on free mass selected species are limited by the low densities of particles. We have thus undertaken since several years [1] to condense mass selected clusters in rare gas matrices in order to study their optical properties. Recently new developments have allowed us to study not only $\mathrm{Ag}$ clusters, but also $\mathrm{Au}$ and $\mathrm{Cu}$ clusters in neon matrices with good signal to noise ratio. Neon matrices have the advantage to be the most inert matrices because of their low polarizability and their higher crystallinity compared to the other rare gas matrices. This helps to diminish undesirable effects, such as electronic level shifting, additional splitting or line broadening.

In particular the bonding in Au clusters, in which relativistic effects manifest strongly, is substantially different from that of silver clusters. Surprisingly, despite several previous studies, neither absorption nor fluorescence spectra of the gold dimer embedded inside a neon matrix have been reported. Furthermore in the visible range, where the main transitions are known to happen from gasphase measurements [2], the existing spectra for $\mathrm{Au}_{2}$ in

\footnotetext{
${ }^{a}$ e-mail: sylvain.lecoultre@epfl.ch

b Present address: Meteoswiss, station aérologique, 1530 Payerne, Switzerland.
}

argon [3-5] or krypton [6] do not report any transition below $3.2 \mathrm{eV}$. In this paper absorption and fluorescence measurements have been obtained in the UV-Visible range for the gold dimer embedded in neon. They are in excellent agreement with the gas-phase measurements.

\section{Experimental setup}

Although the main principles of the measurement procedure are similar to those described in reference [7], we have recently modified the setup in order to improve the control over the deposition of clusters and to reach lower temperatures that allow condensation of neon.

Briefly, the clusters are formed from a metal target sputtered by a $10 \mathrm{~mA} \mathrm{Xe}{ }^{+}$ion beam at $25 \mathrm{keV}$. The gold cations are then focused into a "Bessel Box" type (BB) energy filter which also acts as a beam stop for the intense flux of neutral particles. The cations of interest are massselected and directed towards the sample holder, which consists in a superpolished aluminium mirror (Valley design corp.). The sample holder is fixed to a pulse tube cryostat (SRP-052A Cryocooler, Sumitomo Heavy Industries Ltd.) that allows refrigeration down to $6 \mathrm{~K}$. During sample preparation, the size-selected clusters are accumulated together with the neon matrix for 1 to $1.5 \mathrm{~h}$ while a $50 \mu \mathrm{m}$ thick matrix is grown.

Optical absorption measurements are performed by injecting light through the $2 \mathrm{~mm}$ length of matrix and collecting the residual light on the other side with an optical fiber of $400 \mu \mathrm{m}$ core diameter. The collected light is then analyzed by an optical spectrometer coupled with a liquidnitrogen-cooled charge coupled device (CCD). Comparing the intensity of the light passing through a matrix 
doped with clusters with a reference signal of light passing through a pure neon matrix yields the absorption spectrum.

For excitation, we use a frequency doubled optical parametric oscillator OPO pumped by a Nd:YAG laser. The laser beam is focused onto the sample, which is translated vertically from its initial position in front of a $\mathrm{CaF}_{2}$ window. The fluorescence is collected by the same lens that focuses the laser onto the mirror, in order to form a parallel beam. The collected beam is then focalized in the entrance slit of the spectrometer [8].

\section{Results and discussion}

The electronic properties of gold clusters can be best understood by first looking at the atomic properties of gold. We have therefore performed measurements on the atom and compared them with already existing results in order to test our new experimental setup.

The experimental spectrum of gold atoms in neon is shown in Figure 1. It contains a well defined transition at $4.8 \mathrm{eV}$ with a shoulder at $4.9 \mathrm{eV}$ as well as a larger structure with two maxima between 5.25 and $5.6 \mathrm{eV}$. Comparison with previous measurements in neon [9] and other matrices [5] confirm this spectrum except for the small secondary peak at $4.9 \mathrm{eV}$. This measurement of atomic gold inside a matrix is the first one to show such a sharp transition close to the $4.8 \mathrm{eV}$ one. In other works by Gruen and Bates [9], the neon matrices were systematically annealed at $10 \mathrm{~K}$ and it is possible that such heating of the matrix allows the system to evolve towards a more stable configuration by suppressing metastable sites. Indeed, this secondary peak might be due to the existence of a less favorable second site isomer for the gold atoms inside the neon matrix. This is the case for silver atoms where two site isomers of 3 and 4 vacancies of neon are in competition $[10,11]$. This hypothesis is emphasized by the fact that silver and gold atoms have a very similar size due to relativistic effects that tend to confine the $s$ electrons of gold [12].

Figure 2 shows the absorption spectrum of $\mathrm{Au}_{2}$ in neon. It consists of 3 narrow peaks at $2.58,3.13$ and $3.30 \mathrm{eV}$ and a weak absorption at $3.96 \mathrm{eV}$. Although attention was paid to reduce fragmentation of the dimer by decreasing the deposition energy, it remains difficult to avoid the atom signal. This might be due to the large energy distribution of the dimers that are produced by the sputtering process.

Other measurements recently performed in our group for larger cluster sizes in neon unambiguously confirm these transitions, for instance by looking at the trimer spectrum that includes a large contribution due to the $\mathrm{Au}_{2}$ via fragmentation.

As already mentioned, the gold dimer has been studied in the gas phase $[2,13,14]$, in argon [3-5] and krypton matrices [6]. The gas-phase measurements have shown the presence of two absorption bands at low energies: $\mathrm{A} \leftarrow \mathrm{X}$ around $2.44 \mathrm{eV}$ and $\mathrm{B} \leftarrow \mathrm{X}$ centered around $3.18 \mathrm{eV}$ [15]. With good certainty we can attribute the peak measured

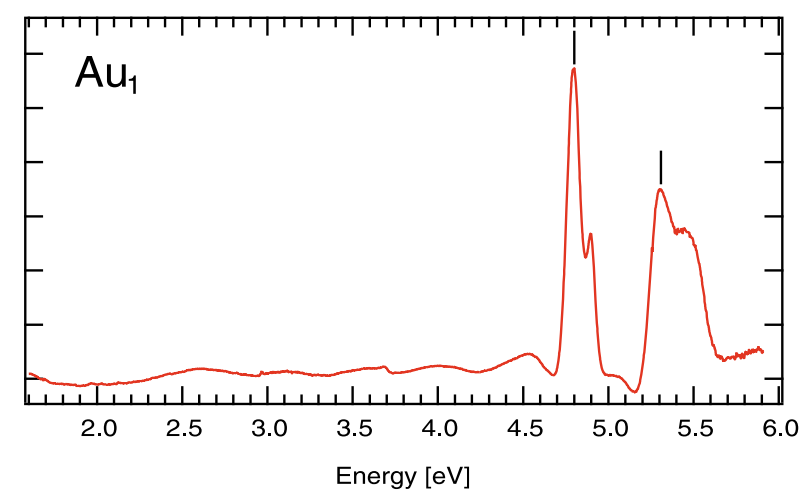

Fig. 1. Absorption spectrum of $\mathrm{Au}_{1}$ in neon at $7 \mathrm{~K}$. The excitation energies for fluorescence (see Fig. 3) are marked by an arrow.

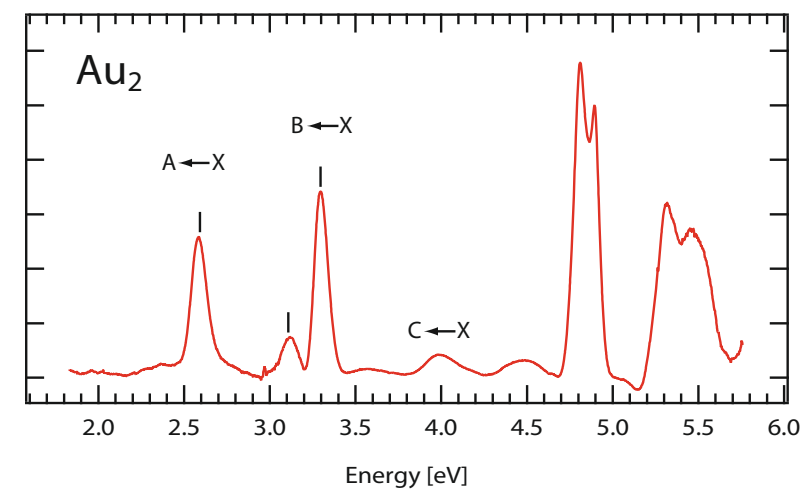

Fig. 2. Absorption spectrum of $\mathrm{Au}_{2}$ in neon at $7 \mathrm{~K}$. The excitation energies for fluorescence (see Fig. 5) are marked by an arrow.

at $2.58 \mathrm{eV}$ to the $\mathrm{A} \leftarrow \mathrm{X}$ absorption measured in the gas phase, and the peak measured at $3.3 \mathrm{eV}$ to the $\mathrm{B} \leftarrow \mathrm{X}$ transition.

Measurements in matrices have found transitions around $3.3-3.5 \mathrm{eV}, 3.9-4.1 \mathrm{eV}$ and $5.9-6 \mathrm{eV}$ depending on the measurement and the type of matrix. To our knowledge however, no other measurement in matrix has ever confirmed the existence of the low energy $\mathrm{A} \leftarrow \mathrm{X}$ transition. The $\mathrm{B} \leftarrow \mathrm{X}$ dimer absorption has been reported by Klotzbücher and Ozin [5] at $3.40 \mathrm{eV}$ and by Fedrigo et al. [4] at $3.47 \mathrm{eV}$ in argon. The weak absorption at $3.96 \mathrm{eV}$, denoted $\mathrm{C} \leftarrow \mathrm{X}$ has also been observed in argon matrices around $4 \mathrm{eV}$ [4], it is however not visible in the gas phase. The symmetry forbidden transition switches on by the interaction with the matrix. The secondary peak at $3.13 \mathrm{eV}$, next to the $\mathrm{B} \leftarrow \mathrm{X}$ transition, has neither been observed in the gas phase, nor in other matrices. Its origin is therefore hard to understand. Notice however in Figure 5 that excitation at 3.13 and $3.30 \mathrm{eV}$ result in the same emission spectra. The relaxation following excitation of the $3.13 \mathrm{eV}$ peak is therefore similar to the $\mathrm{B} \leftarrow \mathrm{X}$ one indicating that this is not due to a second site isomer. 


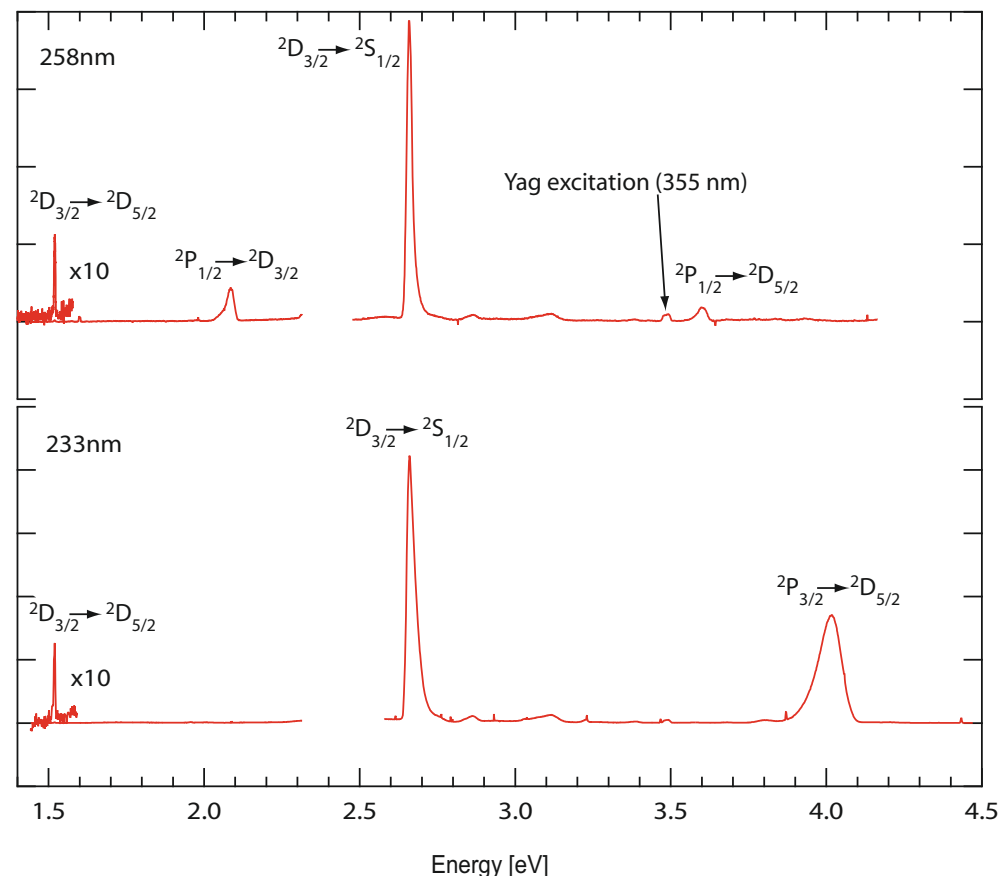

Fig. 3. Fluorescence spectra of $\mathrm{Au}_{1}$ excited at $233 \mathrm{~nm}(5.32 \mathrm{eV})$ and $258 \mathrm{~nm}(4.80 \mathrm{eV})$.

Several calculations have studied the electronic transitions of $\mathrm{Au}_{2}$. In particular recent studies have been done in the framework of the TDDFT scheme [15-17], in which the relativistic corrections are taken in an average sense, i.e. without introducing explicitly the spin-orbit coupling. The calculations show clearly the two major effects of the $d$ electrons on the optical properties of gold clusters, namely the quenching of the oscillatory strength and the strong $s-d$ hybridization in the low energy transitions. Due to the absence of the spin-orbit term, the agreement with the experiment is only partial. These calculations suggest however that an orbital forbidden transition at $2.7 \mathrm{eV}$ [16], could be responsible for the $\mathrm{C} \leftarrow \mathrm{X}$ absorption observed in matrices. More elaborate calculations, that explicitly take into account the spin-orbit coupling [18-20], are in excellent agreement with the $\mathrm{A} \leftarrow \mathrm{X}$ and $\mathrm{B} \leftarrow \mathrm{X}$ transitions observed in the gas phase.

Turning to fluorescence, Figure 3 shows the fluorescence spectra of gold atoms under excitations at $233 \mathrm{~nm}$ $(5.32 \mathrm{eV})$ and $258 \mathrm{~nm}(4.80 \mathrm{eV})$.

Two narrow peaks at 1.52 and $2.66 \mathrm{eV}$ are visible under both excitation wavelengths whereas wider emissions at $4.09 \mathrm{eV}, 3.59 \mathrm{eV}$ and $2.08 \mathrm{eV}$ are only seen for one excitation wavelength. The tiny structures at 2.86 and $3.10 \mathrm{eV}$ are artifacts due to the residual light in the laboratory, while the small structure at $3.49 \mathrm{eV}$ is due to the YAG laser excitation. Existing measurements [21] on the same system assign the peak at $2.66 \mathrm{eV}$ to the ${ }^{2} \mathrm{D}_{3 / 2} \rightarrow{ }^{2} \mathrm{~S}_{1 / 2}$ transition and the one at 1.52 to the ${ }^{2} \mathrm{D}_{3 / 2} \rightarrow{ }^{2} \mathrm{D}_{5 / 2}$ transition while the ${ }^{2} \mathrm{D}_{5 / 2} \rightarrow{ }^{2} \mathrm{~S}_{1 / 2}$ emission line reported at $1.21 \mathrm{eV}$ lies beyond our measurement range. The narrowness of the $\mathrm{D} \rightarrow \mathrm{D}$ transition is due to the localized character of the electronic levels involved [22]. The wider peaks have not been reported so far. We assign the 4.09 eV emis-

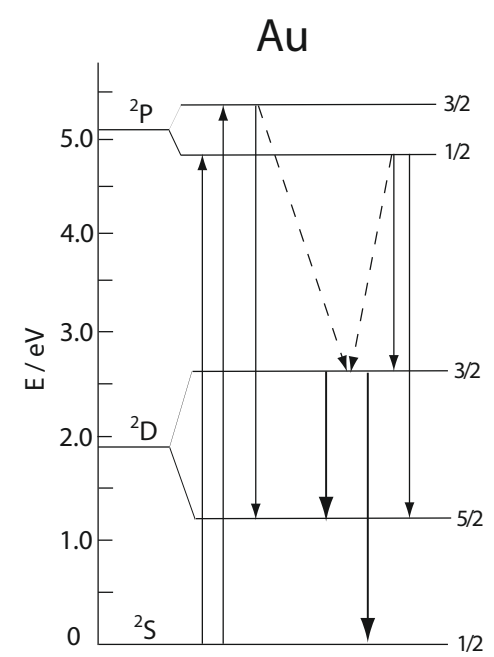

Fig. 4. Energy level diagram of $\mathrm{Au}_{1}$. Five emission lines correspond to the fluorescences observed on the spectra. ${ }^{2} \mathrm{D}_{3 / 2} \rightarrow{ }^{2} \mathrm{D}_{5 / 2}$ at $1.52 \mathrm{eV},{ }^{2} \mathrm{P}_{1 / 2} \rightarrow{ }^{2} \mathrm{D}_{3 / 2}$ at $2.14 \mathrm{eV}$, ${ }^{2} \mathrm{D}_{3 / 2} \rightarrow{ }^{2} \mathrm{~S}_{1 / 2}$ at $2.66 \mathrm{eV},{ }^{2} \mathrm{P}_{1 / 2} \rightarrow{ }^{2} \mathrm{D}_{5 / 2}$ at $3.59 \mathrm{eV}$ and ${ }^{2} \mathrm{P}_{3 / 2} \rightarrow{ }^{2} \mathrm{D}_{5 / 2}$ at $4.09 \mathrm{eV}$.

sion line to the ${ }^{2} \mathrm{P}_{3 / 2} \rightarrow{ }^{2} \mathrm{D}_{5 / 2}$ deexcitation channel. This is in agreement with the observation that it is only present by excitation of the ${ }^{2} \mathrm{P}_{3 / 2}$ level at $5.3 \mathrm{eV}$. The emission lines at $2.08 \mathrm{eV}$ and $3.59 \mathrm{eV}$ are only observed by excitation of the ${ }^{2} \mathrm{P}_{1 / 2}$ state, they are respectively assigned to the ${ }^{2} \mathrm{P}_{1 / 2} \rightarrow{ }^{2} \mathrm{D}_{3 / 2}$ and ${ }^{2} \mathrm{P}_{1 / 2} \rightarrow{ }^{2} \mathrm{D}_{5 / 2}$ transitions. Notice that the low intensity ${ }^{2} \mathrm{P}_{1 / 2} \rightarrow{ }^{2} \mathrm{D}_{5 / 2}$ is not allowed in the gas phase.

Fluorescence measurements of $\mathrm{Au}_{2}$ in neon are given in Figure 5 under excitations at 376 (3.30), 396 (3.13) and $480 \mathrm{~nm}(2.58 \mathrm{eV})$. 


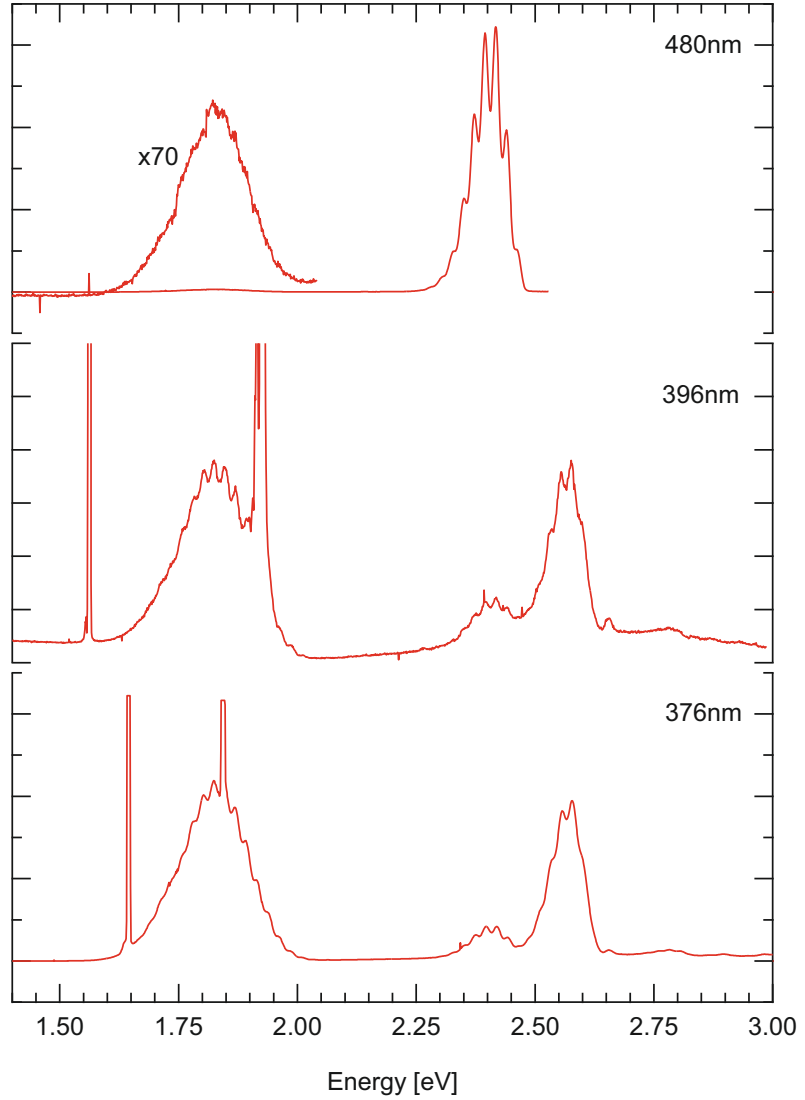

Fig. 5. Fluorescence spectra of $\mathrm{Au}_{2}$ excited at 376 (3.30), 396 (3.13), $480 \mathrm{~nm}(2.58 \mathrm{eV})$. Both narrow peaks at low energies are due to laser excitation.

At least four different emission bands with vibrational resolution have been obtained. They are centered at 1.83 , 2.41 and $2.55 \mathrm{eV}$. A fourth emission, relatively weak but still present at $2.78 \mathrm{eV}$ with a vibrational structure is undoubtedly present. The spectra under excitation at $376 \mathrm{~nm}$ $(3.30 \mathrm{eV})$ and $396 \mathrm{~nm}(3.13 \mathrm{eV})$ are quasi-identical and correspond to the same deexcitation channels. The spectrum resulting from excitation at $480 \mathrm{~nm}(2.58 \mathrm{eV})$ is different with one intense vibrational band that is already present on the other spectra with smaller amplitude. The deexcitation channel around $1.83 \mathrm{eV}$ can also be accessed but its intensity is strongly suppressed by a factor of about 70. It is probable that this fluorescence is resonant with the excitation of the dimer to the A state as the Stoke shift is only $0.13 \mathrm{eV}$. It is interesting to notice that the radiative deexcitation channel at $1.83 \mathrm{eV}$ is mainly visible by excitation of the $\mathrm{B} \leftarrow \mathrm{X}$ transition. This suggests that the A state is very weakly coupled by radiative or matrix assisted relaxation to the state responsible for the $1.83 \mathrm{eV}$ radiative channel.

To our knowledge, these results show for the first time fluorescence spectra of the gold dimer in a neon matrix. Fluorescence of $\mathrm{Au}_{2}$ in argon has been measured by Fedrigo et al. [1] and a large band without any structure has been reported between 1.41 and $1.91 \mathrm{eV}$, which should correspond to the lowest energy structure presented in Figure 5.

The vibrational energy of the ground state deduced from the emission structures goes from $22.9 \mathrm{meV}$ for the lowest energy structure to $22.8 \mathrm{meV}$ and $23.5 \mathrm{meV}$ for the next ones, which is in good agreement with the gas-phase measurement value of $23.7 \mathrm{meV}\left(190.9 \mathrm{~cm}^{-1}\right)$ given by Morse [14].

In this paper, we have reported the absorption spectra of $\mathrm{Au}_{2}$ inside a neon matrix showing the two most intense $\mathrm{A} \leftarrow \mathrm{X}$ and $\mathrm{B} \leftarrow \mathrm{X}$ transitions at 2.58 and $3.30 \mathrm{eV}$. We have also reported new emission lines in the atom fluorescence that correspond well with the already known energy levels in the gold atom. Finally, the fluorescence spectra of the gold dimer resulting from the excitation of the A and B states has been shown. Vibrational energies of the ground state deduced from the emission bands are in good agreement with gas-phase measurements.

We owe warm thanks to Pr. Jean Buttet for insightful discussions and suggestions. This work was founded by the Swiss National Science Foundation.

\section{References}

1. S. Fedrigo, W. Harbich, J. Buttet, Phys. Rev. B 47, 10706 (1993)

2. J. Ruamps, R. Hebd. Séances Acad. Sci. 238, 1489 (1954)

3. S. Fedrigo, W. Harbich, J. Buttet, Int. J. Mod. Phys. B 6, 3767 (1992)

4. S. Fedrigo, W. Harbich, J. Buttet, J. Chem. Phys. 99, 5712 (1993)

5. W.E. Klotzbücher, G.A. Ozin, Inorg. Chem. 19, 3767 (1980)

6. W. Harbich, S. Fedrigo, J. Buttet, J. Chem. Phys. 96, 8104 (1992)

7. F. Conus, J.T. Lau, V. Rodrigues, C. Félix, Rev. Sci. Instrum. 77, 113103 (2006)

8. C. Sieber, Ph.D. thesis, EPFL, 2002

9. D.M. Gruen, J.K. Bates, Inorg. Chem. 16, 2450 (1977)

10. S. Ossicini, F. Forstmann, Inorg. Chem. 21, 1755 (1982)

11. J.T. Zoueu, M. Vala, J.C. Rivoal, Chem. Phys. 312, 89 (2005)

12. D.M. Gruen, M. Moskovits, G.A. Ozin, in Cryochemistry (Wiley-Interscience, New-York, 1976)

13. L.L. Ames, R.F. Barrow, Trans. Faraday Soc. 63, 39 (1967)

14. M.D. Morse, Chem. Rev. 86, 1049 (1986)

15. J.C. Idrobo, W. Walkosz, S.F. Yip, S. Ogut, J. Wang, J. Jellinek, Phys. Rev. B 76, 205422 (2007)

16. X. Wang, X. Wan, H. Zhou, S. Takami, M. Kubo, A. Miyamoto, J. Mol. Struct. 579, 221 (2002)

17. S. Gilb, private communication (2008)

18. W.C. Ermler, Y.S. Lee, K.S. Pitzer, J. Chem. Phys. 70, $293(1978)$

19. I. Itkin, A. Zaitsevskii, Chem. Phys. Lett. 374, 143 (2003)

20. F. Wang, T. Ziegler, E. Lenthe, S. Gisbergen, E.J. Baerends, J. Chem. Phys. 122, 204103 (2005)

21. W. Schrittenlacher, D.M. Kolb, Ber. Bunsenges. Phys. Chem. 88, 492 (1984)

22. W. Schrittenlacher, H.H. Rotermund, W. Schroeder, D.M. Kolb, Surf. Sci. 156, 777 (1985). 